\title{
Bicentennial Decrease of the Total Solar Irradiance Leads to Unbalanced Thermal Budget of the Earth and the Little Ice Age
}

\author{
Habibullo I. Abdussamatov \\ Pulkovo Observatory of the RAS \\ Pulkovskoye shosse 65, St. Petersburg, 196140, Russia \\ Email: abduss@gao.spb.ru
}

Received: September 22, 2011 Accepted: October 9, $2011 \quad$ Published: February 1, 2012

doi:10.5539/apr.v4n1p178

URL: http://dx.doi.org/10.5539/apr.v4n1p178

\begin{abstract}
Temporal changes in the power of the longwave radiation of the system Earth-atmosphere emitted to space always lag behind changes in the power of absorbed solar radiation due to slow change of its enthalpy. That is why the debit and credit parts of the average annual energy budget of the terrestrial globe with its air and water envelope are practically always in an unbalanced state. Average annual balance of the thermal budget of the system Earth-atmosphere during long time period will reliably determine the course and value of both an energy excess accumulated by the Earth or the energy deficit in the thermal budget which, with account for data of the TSI forecast, can define and predict well in advance the direction and amplitude of the forthcoming climate changes. From early 90s we observe bicentennial decrease in both the TSI and the portion of its energy absorbed by the Earth. The Earth as a planet will henceforward have negative balance in the energy budget which will result in the temperature drop in approximately 2014. Due to increase of albedo and decrease of the greenhouse gases atmospheric concentration the absorbed portion of solar energy and the influence of the greenhouse effect will additionally decline. The influence of the consecutive chain of feedback effects which can lead to additional drop of temperature will surpass the influence of the TSI decrease. The onset of the deep bicentennial minimum of TSI is expected in $2042 \pm 11$, that of the $19^{\text {th }}$ Little Ice Age in the past 7500 years - in $2055 \pm 11$.
\end{abstract}

Keywords: TSI decrease, Little Ice Age

\section{Introduction}

William Herschel (1801) was the first to report correlation between a level of sunspot activity and a climate after his discovery of inverse interrelation between a wheat price and a level of cyclic variations of solar activity before and during Dalton minimum. When the Sun's surface was covered with sunspots, the wheat prices were going down. When the number of sunspots dropped the prices went up. He supposed that variations of wheat prices are the consequence of the corresponding climate changes. However, he could not explain the physical nature of this phenomenon. Later Eddy (1976) was discovered interconnection between clearly determined periods of significant variations of the sunspot activity level during the last millennium and corresponding deep climatic changes in both phase and amplitude. During each of the eighteen deep minima of solar activity (of Maunder type) with a bicentennial cycle found in the preceding 7.5 millennia, deep cooling was observed, while during the periods of high maxima - global warming (Borisenkov, 1988). Recent studies (Bal, et al. 2011; McPhaden, et al. 2011) confirm our results (Abdussamatov, 2009a, b) concerning a common action of eleven-year and bicentennial cyclic variations of the total solar irradiance (TSI) (with some time-lag) on the change of state of the surface and subsurface layers (with the depth of tens and hundreds of meters) in the tropical part of the Pacific Ocean accompanied with appearance of warm or cold water (the cycles of La Niña or El Niño phenomena) which affects the climate change as well. Observed characteristics of El Niño during the last 31 years have been changing in the opposite direction with regard to predictions of the climatic models assuming predominant influence of the greenhouse gases.

\section{Variation of the TSI and Secondary Feedback Effects}

Both bicentennial and eleven-year cyclic variations of TSI and solar activity are synchronized and inter-correlated in both phase and amplitude (Fig. 1) (Abdussamatov, 2004, 2005, 2007a, b). This allows to use and extrapolate a relatively short (since 1978) series of precise measurements of TSI (Fröhlich, 2011)), 
comparing them with existing long series of solar activity data (Shapiro et al., 2011). Hence, all periods of significant climate changes found during the last 7,500 years were caused by bicentennial quasi-periodic TSI variations. However, direct influence of relatively small bicentennial fluctuation of TSI (according to recent reconstructed data (Shapiro et al., 2011) - of the order of $0.5 \% \approx 6.8 \mathrm{~W} / \mathrm{m}^{2}$ ) is insufficient to explain corresponding cyclic bicentennial temperature variations on the Earth from global warming to the Little Ice Ages. We need a kind of amplifier of direct influence of TSI variations on observed climate changes. The role of such amplifier can play TSI secondary additional influence in a form of feedback effects: natural change of the Earth's global albedo, Bond albedo, and of the atmospheric concentration of greenhouse gases (first of all, water vapor and also carbon dioxide, methane and other gases). Bond albedo is determined by three global optical parameters of the whole globe with its air and water envelopes averaged along a whole vertical line surface-atmosphere: by the values of both the atmospheric albedo and albedo of the Earth surface, as well as by the value of atmospheric transmission in the solar spectrum. Thus, Bond albedo is one of the main physical parameters in the energy budget of the Earth as a planet. Its (albedo) significant changes could be potential powerful engine climate variations. The value of the Earth's albedo is rising up to a maximally high level during a deep cooling and drops down to a minimal level in the process of global warming while variations of the greenhouse gases atmospheric concentration take place in an opposite direction since their abundance is mostly determined by the temperature of the World Ocean. Variations of characteristics of both the surface of the Earth and its atmosphere caused by bicentennial variations of TSI give birth to a subsequent chain of further additional temperature changes caused by multiple repetition of this cause-and-effect cycle of secondary feedback effects even if TSI remains constant for a certain period of time. The influence of the consecutive chain of such changes caused by secondary feedback effects can result in additional amplification of the climatic changes to the extent exceeding the influence of bicentennial TSI variations. Similar picture was observed at the end of the XXth century.

\section{Energy Budget of the System Earth-atmosphere}

Temporal changes in the power of the longwave radiation of the system Earth-atmosphere emitted to space always lag behind changes in the power of absorbed solar radiation due to slow change of its enthalpy. Thermodynamic planetary temperature determining the integral heat balance of the planet changes with significant time-lag with regard to the process of changes in the power of absorbed solar radiation depending on the thermal inertia of the system Earth-atmosphere. This is equivalent to excess or deficit of the budget of absorbed and emitted power. Any long-term fluctuations of the solar energy absorbed by the Earth due to bicentennial variation of TSI with account for slow changes in the enthalpy of the system Earth-atmosphere during a time period determined by the thermal inertia remain uncompensated by the emission of the intrinsic longwave radiation to space. This process is described by the increment of the thermodynamic planetary temperature slowly changing with time. That is why an average annual balance of the energy budget of the Earth as a planet is practically always in an unbalanced state and oscillates around an equilibrium state absorbing and emitting different amount of energy due to bicentennial variation of TSI. As a result, the planet will either get warm or cool down. Average annual difference between the energy of solar radiation entering the outer layers of the Earth atmosphere and both a fraction of this energy reflected to space and the energy of longwave radiation determines the balance of thermal budget of the system Earth-atmosphere. Specific power of the enthalpy change for the system Earth-atmosphere - a difference between incoming and outgoing radiation $E$ is descried by the equation:

$$
E=\left(S_{\odot}+\Delta S_{\odot}\right) / 4-(A+\Delta A)\left(S_{\odot}+\Delta S_{\odot}\right) / 4-\varepsilon \sigma\left(T_{p}+\Delta T_{p}\right)^{4} ; \quad E=C d T_{p} / d t
$$

where $S_{\odot}$ is the TSI, $\Delta S_{\odot}$ - increment of TSI, $A$ - Earth global albedo (Bond albedo), $\Delta A$ - Bond albedo increment, $\varepsilon$ - emissivity of the system Earth-atmosphere, $\sigma$-Stefan-Boltzmann constant; $T_{p}$ - thermodynamic temperature of the planet, $E$ - specific power of the enthalpy change for the active layer of the atmosphere and the Ocean $\left(\mathrm{W} / \mathrm{m}^{2}\right), C$ - specific surface heat capacity of the active layer of the atmosphere and the Ocean, with regard to the total surface of the planet $\left(\mathrm{J} / \mathrm{M}^{2} \mathrm{~K}\right), t-$ time. $1 / 4$ in the right part of the equation reflects the fact that the flux of solar radiation is projected (on the circle) and reflected from the circle, while the Earth emits from the total surface of the sphere (which is four times larger). Specific power of the enthalpy change of the Earth, $E$, denotes the deficit or excess of thermal energy which can be regarded as energetic balance of the average annual budget in the debit and credit of the thermal power of the planet.

At the same time increment of the Earth effective temperature determining the radiative balance occurs immediately with the change of absorbed power in contrast to the planetary thermodynamic temperature 
determining the thermal balance. Relative influence of variations of the Bond albedo and TSI on the change of the Earth effective temperature can be determined on the basis of the radiative balance of the Earth as a planet:

$$
S_{\mathscr{\sigma}} 4=\sigma T_{e}^{4}+A S_{\mathscr{\sigma}} 4
$$

where $T_{e}-$ is the effective temperature of the Earth.

Let us introduce an increment of the Earth effective temperature $\Delta T_{e}=T_{e}-T_{e o}$, where $T_{e}$ is a current value of effective temperature, $T_{e o}$ - its initial value. We consider this increment to be caused by the increments of TSI, $\Delta S_{\odot}$, and of Bond albedo, $\Delta A$. In this case:

$$
\Delta S_{\odot} / 4=4 \sigma T^{3} e_{e} T_{e}+A \Delta S_{\odot} / 4+\Delta A\left(S_{\odot}+\Delta S_{\odot}\right) / 4
$$

From (3) one can get an expression for the increment of the Earth effective temperature caused by the increments of TSI and of Bond albedo:

$$
\Delta T_{e}=\left[\Delta S_{\odot}(1-A-\Delta A)-\Delta A S_{\Theta}\right] / 16 \sigma T_{e}^{3} .
$$

For constant TSI $\left(\Delta S_{\odot}=0\right)$ we can obtain from (4):

$$
\Delta T_{e}=-\Delta A S_{\sigma} 16 \sigma T^{3}{ }_{e}
$$

Taking into account the known values of the Earth effective temperature whose current value is $T_{e}=254.8 \mathrm{~K}$ and TSI $-S_{\odot}=1366 \mathrm{~W} / \mathrm{m}^{2}$, and putting $\Delta S_{\odot}=0$ we can derive from (5):

$$
\Delta T_{e}=-91 \Delta A .
$$

For constant Bond albedo $\Delta A=0$ we get from (4):

$$
\Delta T_{e}=\Delta S_{\odot}(1-A) / 16 \sigma T_{e}^{3} .
$$

Using a known value of the Earth global albedo which according to recent data is $A=0.30$ (Trenberth et al., 2009) and $\Delta A=0$ we obtain from (7):

$$
\Delta T_{e}=0.047 \Delta S_{\odot}
$$

Evaluation of relative contribution of the increments $\Delta S_{\odot}$ and $\Delta A$ to the increment $\Delta T_{e}$ can be done by taking

$$
\Delta S_{\odot}(1-A-\Delta A)-\Delta A S_{\odot}=0
$$

in Formula (4). From (9) one can get the ratio of relative contribution of the increments $\Delta S_{\odot}$ and $\Delta A$ to the increment $\Delta T_{e}$

$$
\Delta S_{\odot} S_{\odot}=\Delta A /(1-A-\Delta A)
$$

or

$$
\Delta S_{\odot}=1366 \cdot \Delta A /(0.7-\Delta A)
$$

The increase of the Bond albedo by $\triangle A=+0.003(1.0 \%)$ will result in decrease of the effective temperature by $\Delta T \approx-0.27 \mathrm{~K}$, which is practically equivalent to bicentennial decrease of the TSI value $\Delta S_{\odot}=-5.88 \mathrm{~W} / \mathrm{m}^{2}$ $(0.435 \%)$.

Thus, the long-term change of the Bond albedo is a powerful force for variations of the Earth climate.

Decrease of TSI by $0.5 \% \Delta S_{\odot}=-6.83 \mathrm{~W} / \mathrm{m}^{2}$ with constant Bond albedo, $\Delta A=0$, leads according to (8) to decrease of the effective temperature of the whole Earth with its air and water envelopes by $\Delta T_{e}=-0.32 \mathrm{~K}$ (the difference between changes in temperature of global surface air with time-lag and radiation is insignificant). Decrease of the effective temperature of the Earth by $\Delta T_{e}=-0.32 \mathrm{~K}$, following (6), can cause increase of the Earth global albedo by $\Delta A=+0.0035$ or by $1,16 \%$. With such increase of the Bond albedo by $1.16 \%$ the effective temperature of the Earth as a planet will additionally decrease by $\sim 0.3 \mathrm{~K}$, which results in a chain of such cycles. However, the effective (radiative) temperature of the system Earth-atmosphere describes inertia-free process of radiative heat exchange in the equilibrium thermal regime. Due to this the instant radiative balance is realized with advance relative to total energetic (or heat) balance of the planet - formula (1) with account for slow changes the enthalpy of the system Earth-atmosphere. Effective temperature is a radiative temperature of 
the planet and does not reflect temporal variations of the planetary temperature, but indicates the tendencies in direction of the planet climate change. Thus, the change of the Bond albedo value significantly affects changes in the effective (radiative) temperature of the Earth thus being one of the most important factors determining forthcoming climate changes. However, changes of the thermodynamic temperature of the Earth due to variations of the Bond albedo and TSI do not occur instantly but with significant time-lag, determined by the thermal inertia of the planet (Abdussamatov et al., 2010).

$$
t=0.095(1+0.42 \cdot l) y r
$$

where $l-$ is the depth of the active layer of the Ocean. If the depth of its active layer is about $200-500 \mathrm{M}$, the thermal inertia is:

$$
t=14 \pm 6 y r .
$$

Due to quite large heat capacity of the Ocean the thermodynamic temperature of the planet (with account for emissivity) changes rather slowly. Hence the debit and credit parts of the average annual energy budget of the terrestrial globe with its air and water envelope are practically always in an unbalanced state $(E \neq 0)$, having either positive or negative balance. Such unbalanced average annual thermal budget is a basic state of climatic system Earth-atmosphere. During long-term decline of TSI the average annual enthalpy change in the system Earth-atmosphere turns out to be negative $(E<0)$, while during long-term increase of TSI - positive $(E>0)$. At the same time variations of TSI and Bond albedo play the most important role in the change of both the energy balance of the system Earth-atmosphere and its thermodynamic temperature. Average annual balance of the thermal budget of the system Earth-atmosphere during long time period will reliably determine the course and value of both an energy excess accumulated by the Earth or the energy deficit in the thermal budget and, with account for data of the TSI forecast, can define for certain and predict well in advance the direction $(\Delta \mathrm{E}>0$ leads to warming up, $\Delta \mathrm{E}<0$ - to cooling down) and amplitude of the forthcoming changes in global climate.

\section{Bicentennial Decrease of the TSI Leads to the Little Ice Age}

From early 1990s the values of both eleven-year and bicentennial components of TSI variations are decreasing at accelerating (at present) rate (Fig. 2), and hence a fraction of TSI absorbed by the Earth is declining at practically the same rate (e.g., Fröhlich, 2011; Abdussamatov, 2007b, 2009a, b). Average value of TSI in the $23^{\text {rd }}$ cycle was by $0.17 \mathrm{~W} / \mathrm{m}^{2}$ less than in the $22^{\text {nd }}$ cycle. Smoothed value of TSI in the minimum between the cycles $23 / 24$ $\left(1365.24 \pm 0.02 \mathrm{~W} / \mathrm{m}^{2}\right)$ was by $0.26 \mathrm{~W} / \mathrm{m}^{2}$ and by $0.33 \mathrm{~W} / \mathrm{m}^{2}$ less than in the minima between cycles $22 / 23$ and 21/22, respectively. However, forming from early 1990s long-term deficit of TSI (see Fig. 2) was not compensated by decrease in the emission of the Earth intrinsic thermal energy into space which practically remains on the same high level during $14 \pm 6$ years due to thermal inertia of the World Ocean. Since the Sun is now entering a bicentennial long-term phase of low luminosity (e.g., Abdussamatov, 2004, 2005, 2007b; Penn and Livingston, 2010; American-astronomical-society, 2011) such energy imbalance of the system $(\mathrm{E}<0)$ will continue further for the next few 11-year cycles. As a result, the Earth as a planet will henceforward have negative balance $(E<0)$ in the energy budget. This gradual consumption of solar energy accumulated by the World Ocean during the whole XX century will result in decrease of global temperature after $14 \pm 6$ years because of a negative balance in the energy budget of the Earth. This, in its turn, will lead to the rise of Earth albedo, the drop of atmospheric concentration of the most important greenhouse gas - water vapor, as well as of carbon dioxide and other gases. Let us note that water vapor absorbs $\sim 68 \%$ of the integral power of the intrinsic long-wave emission of the Earth, while carbon dioxide - only $\sim 12 \%$. As a consequence, a portion of solar radiation absorbed by the Earth will gradually go down together with manifestations of the greenhouse effect caused by the secondary feedback effects. The influence of the growing consecutive chain of such changes will cause additional decrease of the global temperature exceeding the effect of a bicentennial TSI decrease.

Since the Sun is now approaching the phase of decrease of bicentennial luminosity on the basis of observed accelerating drop in both the 11-year and bicentennial components of TSI from early 90s, we can forecast its further decline similar to a so called Maunder minimum down to $1363.4 \pm 0.8 \mathrm{~W} / \mathrm{m}^{2}, 1361.0 \pm 1.6 \mathrm{~W} / \mathrm{m}^{2}$ and down to a deep minimal level $1359.5 \pm 2.4 \mathrm{~W} / \mathrm{m}^{2}$ in the minima between the cycles $24 / 25,25 / 26$ and $26 / 27$, respectively (Fig. 3). Assuming an expected increase in the duration of the eleven-year cycles during the phase of decline of a bicentennial cycle (Abdussamatov, 2006, 2009a,b), we can expect the approximate moment of minimum between the cycles $24 / 25,25 / 26$ and $26 / 27$ in $2020.3 \pm 0.6,2031.6 \pm 1.2$ and $2042.9 \pm 1.8$, respectively. Under these circumstances the maximal smoothed for 13 months level of sunspot number in the cycles 24,25 and 26 can reach $65 \pm 15,45 \pm 20$ and 30 \pm 20 , respectively (Abdussamatov, 2007b, 2009a,b). Hence, we can expect the onset 
of a deep bicentennial minimum of TSI in approximately $2042 \pm 11$ and of the $19^{\text {th }}$ deep minimum of global temperature in the past 7500 years - in $2055 \pm 11$ (Fig. 4). In the nearest future we will observe a transition (between global warming and global cooling) period of unstable climate changes with the global temperature fluctuating around its maximum value reached in 1998-2005. After the maximum of solar cycle 24, from approximately 2014 we can expect the start of the next bicentennial cycle of deep cooling with a Little Ice Age in 2055 \pm 11 . Thus, long-term variations of TSI (with account for their direct and secondary, based on feedback effects, influence) are the main fundamental cause of climate changes since variations of the Earth climate is mainly determined by a long-term imbalance between the energy of solar radiation entering the upper layers of the Earth's atmosphere and the total energy emitted from the Earth back to space.

\section{References}

Abdussamatov H. I. (2004). About the long-term coordinated variations of the activity, radius, total irradiance of the Sun and the Earth's climate. Proceedings of IAU Symposium, No. 223, Cambridge university press, 541-542.

Abdussamatov H. I. (2006). The time of the end of the current solar cycle and the relationship between duration of 11-year cycles and secular cycle phase. Kinematics and Physics of Celestial Bodies, 22, 141-143.

Abdussamatov H. I. (2007a). On decreasing a total irradiance and downturn of the global temperature of the Earth up to a global cooling in the middle XXI centuries. Bulletin of the Crimean Astrophysical Observatory, 103, No. 4, 292-298.

Abdussamatov H. I. (2007b). Optimal prediction of the peak of the next 11-year activity cycle and of the peaks of several succeeding cycles on the basis of long-term variations in the solar radius or solar constant. Kinematics and Physics of Celestial Bodies, 23, 97-100.

Abdussamatov H. I. (2009a). The Sun defines the climate. Russian journal "Nauka i Zhizn" ("Science and Life"), No 1, pp. 34-42 http://www.gao.spb.ru/english/astrometr/abduss_nkj_2009.pdf

Abdussamatov H. I. (2009b). The Sun dictates the climate, St Petersburg, 197 p. In Russian by Logos publishers.

Abdussamatov H. I. (2005). Long-term variations of the integral radiation flux and possible temperature changes in the solar core. Kinematics and Physics of Celestial Bodies, 21, 328-332.

Abdussamatov H. I., A. I. Bogoyavlenskii, S. I. Khankov, \& Y. V. Lapovok. (2010). Modeling of the Earth's planetary heat balance with electrical circuit analogy. J. Electromagnetic Analysis \& Applications, 2, 133-138.

American-astronomical-societ (2011).

$\mathrm{http}: / / w w w . m y w e a t h e r t e c h . c o m / 2011 / 06 / 14 /$ american-astronomical-society-joins-the-dark-side/

http://cbdakota.wordpress.com/2011/06/24/\%E2\%80\%9Ccheshire-cat-sunspots\%E2\%80\%9D-livingston-and-pe $\mathrm{nn} /$

Bal S. et al. (2011). On the robustness of the solar cycle signal in the Pacific region. Geophysical research letters, 38, L14809,-5 pp.

Borisenkov E. P. (1988). Climate variations during the last millennium. Leningrad, (in Russian).

Eddy J. A. (1976). The Maunder Minimum. Science, 192, 1189-1202.

Fröhlich C. (2011). Solar Constant www.pmodwrc.ch/pmod.php?topic=tsi/composite/SolarConstant

Herschel W. (1801). Observations tending to investigate the nature of the Sun, in order to find the causes or symptoms of its variable of light and heat; with remarks on the use that may possibly be drawn from solar observations. Phil. Trans. Roy. Soc, London, 91, 265-318.

McPhaden M. J. et al. (2011). El Nino and its relationship to changing background conditions in the tropical Pacific Ocean. Geophysical research letters, 38, 115709, 4 pp.

Penn M., \& W. Livingston. (2010). Long-Term Evolution of Sunspot Magnetic Fields. [Online] Available: arXiv:1009.0784v1 [astro-ph.SR]; Link to raw data

Shapiro A. I. et al. (2011). A new approach to the long-term reconstruction of the solar irradiance leads to large historical solar forcing. Astronomy and Astrophysics, 529, A67.

SIDC - Solar Influences Data Analysis Center. (2011). http://sidc.oma.be/sunspot-data/

Trenberth K.E., J.T. Fasullo, \& J. Kiehl. (2009). Earth's global energy budget. Bull. Amer. Meteor. Soc., 90, No. 3, 311-324. 


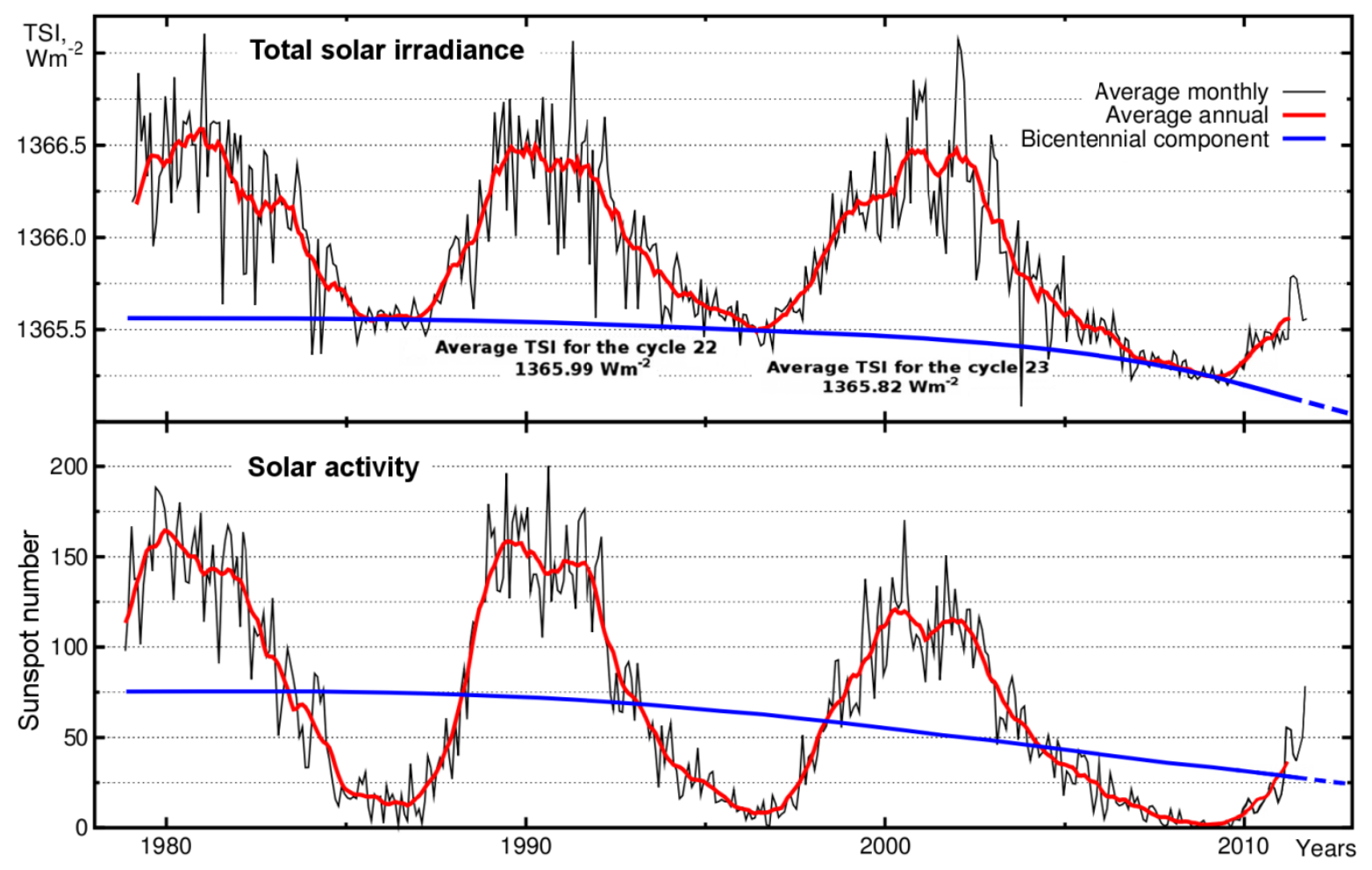

Figure 1. Variations of both the TSI (daily data have been taken from [Fröhlich, 2011]) and solar activity (monthly data have been taken from [SIDC, 2011]) in 1978-2011

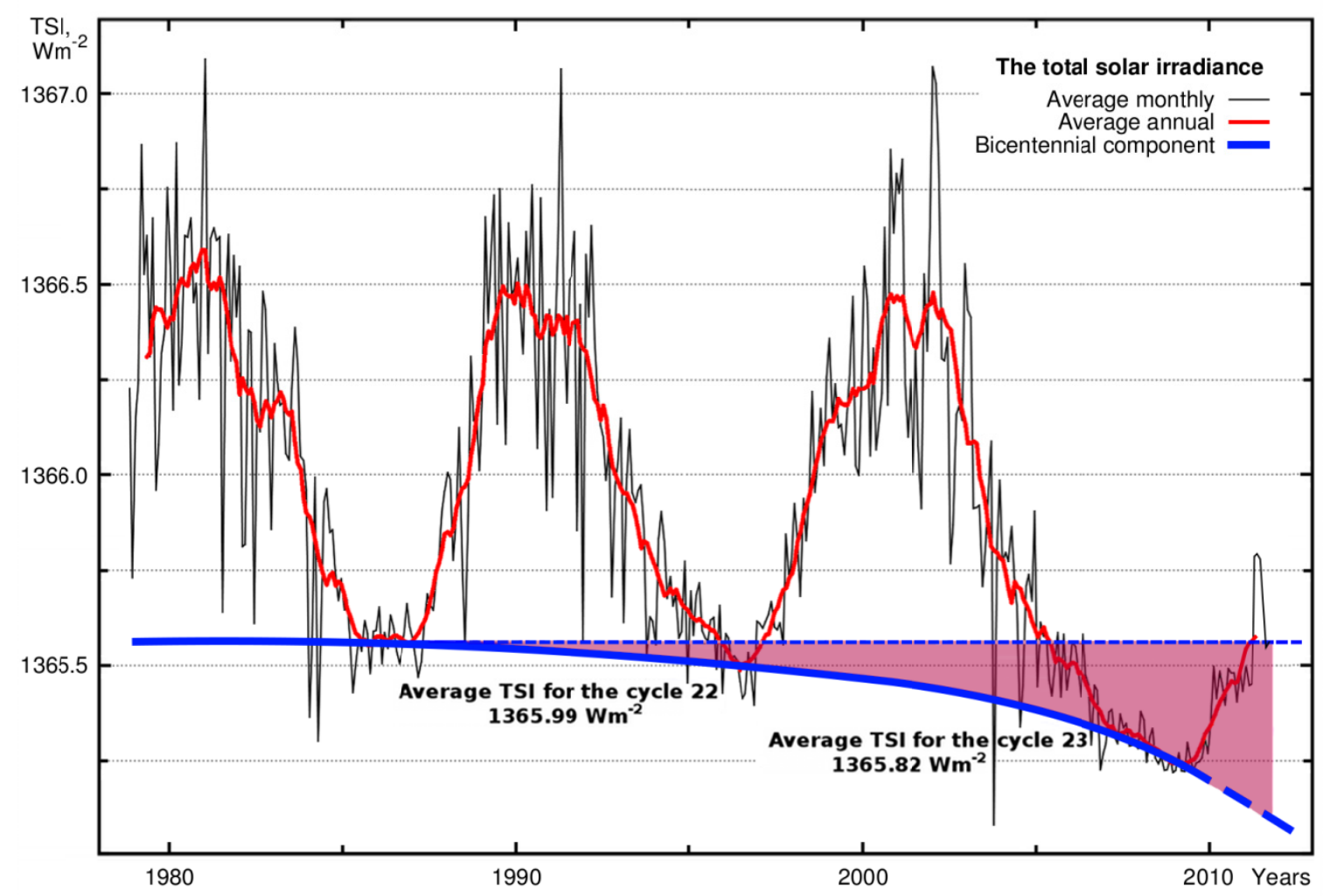

Figure 2. Variations of the TSI in 1978-2011 and deficit of the TSI since 1990 


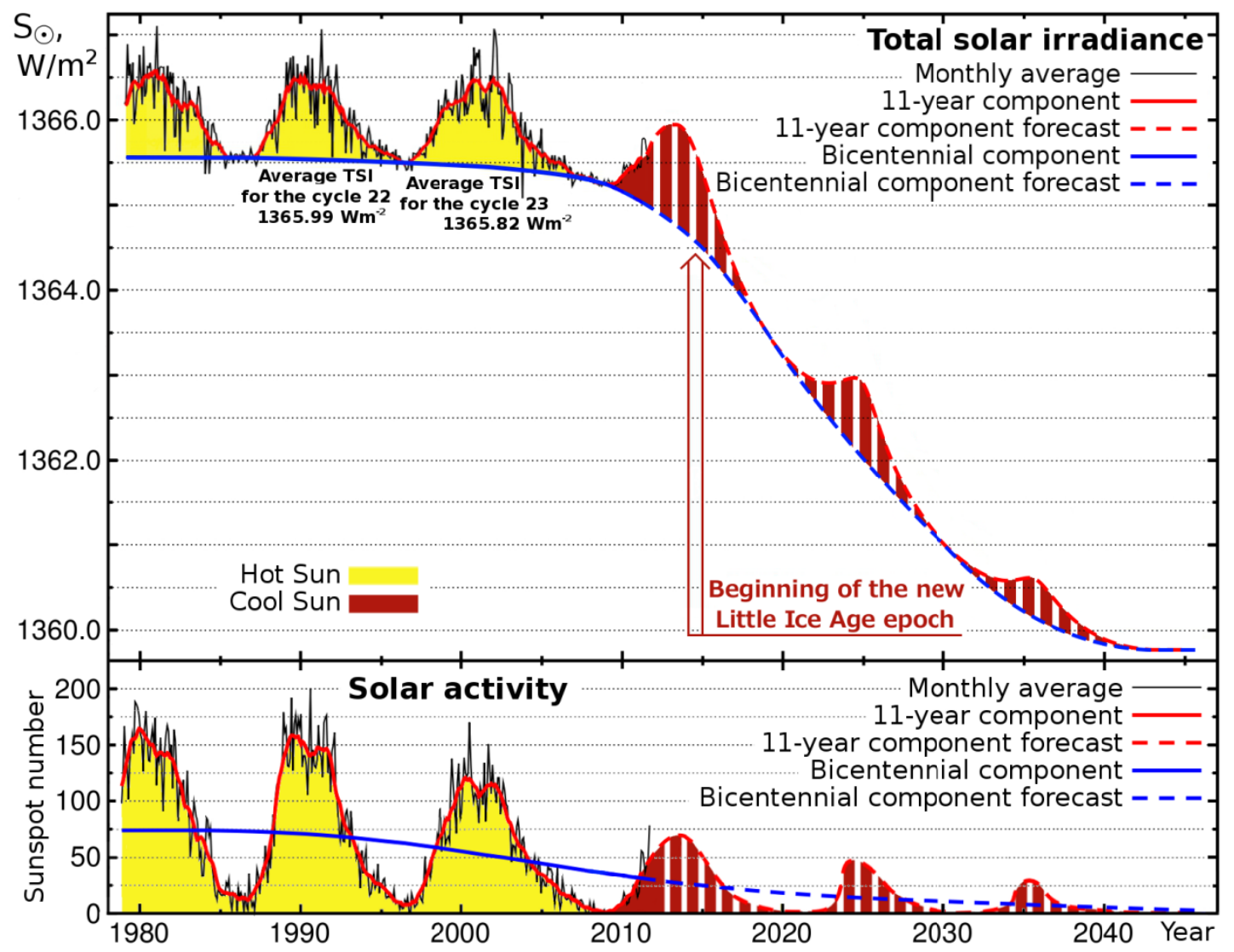

Figure 3. Variations of both the TSI and solar activity in 1978-2011 and a forecast of their variations in cycles 24-26 (up to the year 2045)

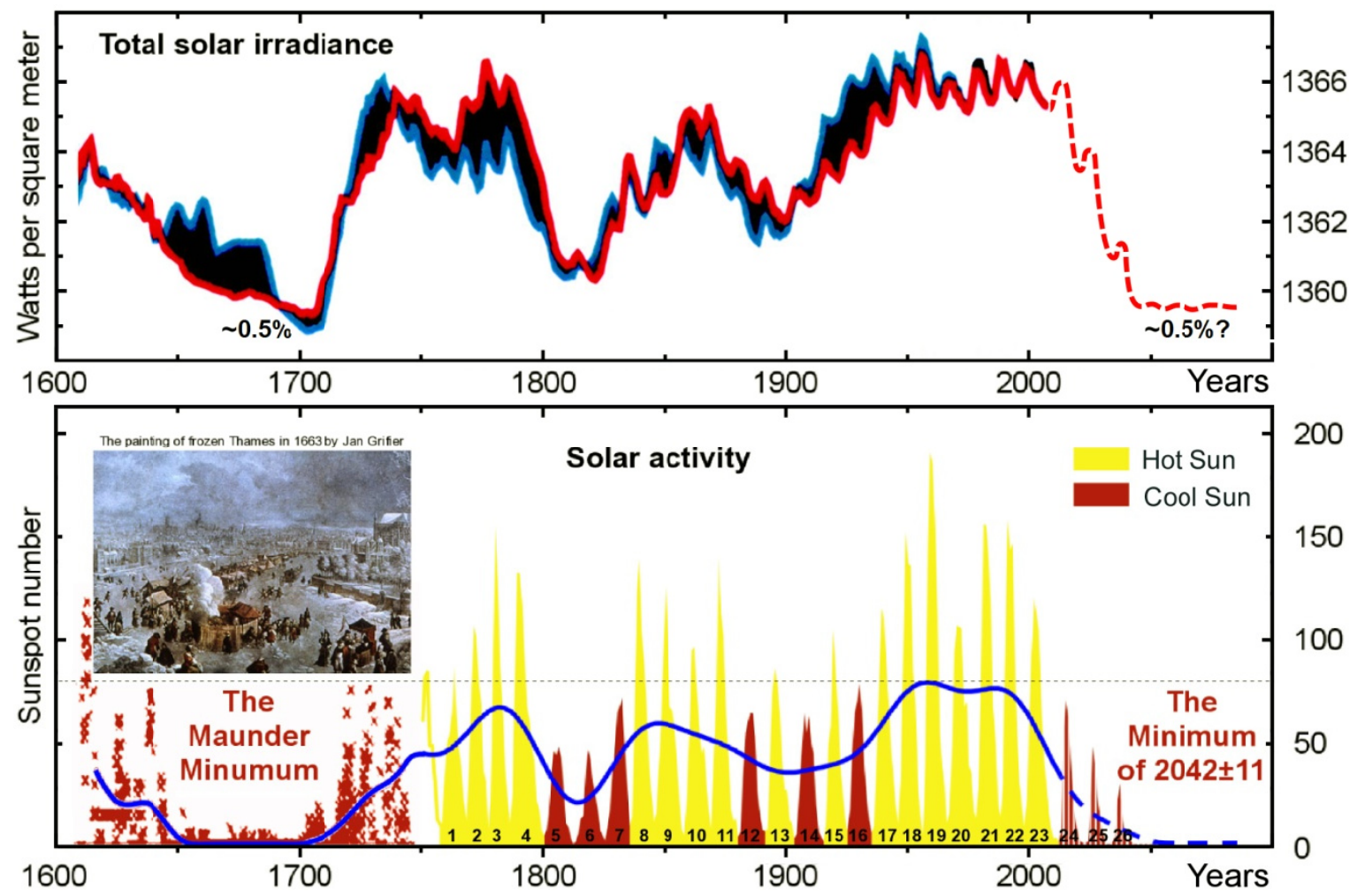

Figure 4. The TSI and solar activity variations since 1611 and our forecast their changes (dash lines) 